\title{
Pitch Matching between Electrical Stimulation of a Cochlear Implant and Acoustic Stimuli Presented to a Contralateral Ear with Residual Hearing
}

\author{
Chin-Tuan Tan ${ }^{*}$, Brett Martin ${ }^{\dagger}$, and Mario A. Svirsky ${ }^{*}$ \\ *Department of Otolaryngology, NYU School of Medicine, New York, NY \\ †Speech and Hearing Sciences, Graduate Center, City University of New York, New York, NY
}

\begin{abstract}
Background-Cochlear implants (CIs) successfully restore hearing in postlingually deaf adults, but in doing so impose a frequency-position function in the cochlea that may differ from the physiological one.

Purpose-The CI-imposed frequency-position function is determined by the frequency allocation table programmed into the listener's speech processor and by the location of the electrode array along the cochlea. To what extent can postlingually deaf CI users successfully adapt to the difference between physiological and CI-imposed frequency-position functions?
\end{abstract}

Research Design-We attempt to answer the question by combining behavioral measures of electroacoustic pitch matching (PM) and measures of electrode location within the cochlea.

Study Sample-The participants in this study were 16 adult CI users with residual hearing who could match the pitch of acoustic pure tones presented to their unimplanted ears to the pitch resulting from stimulation of different $\mathrm{CI}$ electrodes.

Data Collection and Analysis-We obtained data for four to eight apical electrodes from 16 participants with CIs (most of whom were long-term users), and estimated electrode insertion angle for 12 of these participants. PM functions in this group were compared with the two frequency-position functions discussed above.

Results-Taken together, the findings were consistent with the possibility that adaptation to the frequency-position function imposed by CIs does happen, but it is not always complete.

Conclusions-Some electrodes continue to be perceived as higher pitched than the acoustic frequencies with which they are associated despite years of listening experience after cochlear implantation.

\section{Keywords}

auditory adaptation; cochlear implant; electric-to-acoustic pitch matching 


\section{INTRODUCTION}

Cochlear implants (CIs) have been very successful clinically, due in part to the way they mimic the tonotopic organization of the cochlea. In a normal-hearing individual, lower frequency sounds stimulate cochlear locations that are closer to the apex and higher frequency sounds stimulate cochlear locations closer to the base. CI speech processors analyze the incoming acoustic signals into frequency bands and stimulate more apical electrodes in response to lower frequencies and basal electrodes in response to higher frequencies. However, in postlingually deaf patients there are no guarantees that a given input acoustic frequency will result in stimulation of neurons with a characteristic frequency (CF) that matches the frequency of the input. To the extent that pitch percepts are driven by place of stimulation along the cochlea, there may be a mismatch between the pitches elicited by the same input frequency before and after implantation: in other words, a mismatch between the pitch elicited by the $\mathrm{CI}$ in response to a given acoustic frequency and the pitch that was elicited by the same frequency when the listener had normal hearing. The CF of the stimulated neurons could be higher, similar, or lower than the input frequency, depending on factors such as the location of each electrode in the cochlea, the geometry of the electrical fields created by the implant (and the population of neurons that are ultimately activated by those fields), and the mapping between input acoustic frequency and electrode number.

In postlingually deaf CI users, initial pitch matching (PM) may reflect their tonotopic cochlear organization in response to acoustic hearing as classically represented by Greenwood's equation (Greenwood, 1990), originally derived to estimate CF along the organ of Corti (OC). However, CIs stimulate neurons in the spiral ganglion (SG) rather than in the OC. Thus, it is necessary to correct Greenwood's equation to account for the fact that nerve bundles from the $\mathrm{OC}$ in the middle and apical turns take a trajectory into the modiolus that deviates significantly from a radial direction. This correction is provided by the SG frequency-position function measured by Stakhovskaya et al (2007). In any case, and subject to individual differences in neural survival, we might expect that PM of electrodes immediately after implantation might approximate the SG frequency-position function. This contrasts with the frequency-position function imposed by the CI, which is determined by the frequency allocation table (FAT) programmed into the listener's speech processor and by the location of the electrode array along the cochlea.

To what extent can postlingually deaf CI users successfully adapt to the difference between predeafening and postimplantation frequency-position functions? We know from CI users' subjective comments and from pitch-scaling (Fitzgerald et al, 2008) and pitch-ranking experiments (Svirsky et al, 2001; Vermeire et al, 2013) that CI stimulation does produce largely tonotopic percepts, but we do not really know what CI stimulation sounds like, other than what we can glean from the listeners' qualitative descriptions. Direct matching of the pitch perceived in response to stimulation of intracochlear electrodes to the pitch elicited by an acoustic frequency was not possible until the appearance of CI users who had residual acoustic hearing in the contralateral ear. Most often, these individuals, known as bimodal CI users because they are listening with both electric and acoustic modalities, have usable lowfrequency hearing in their unimplanted ear (Toner et al, 2004). Here we use PM data, combined with electrode location estimates, to answer the question posed above and to 
determine the limits (if any) of human capacity to adapt to a change in peripheral sensory input. In individuals who have adapted completely, we would expect that their PM results will closely follow the FAT curve. In other words, if a given electrode is associated with a filter whose center frequency is $500 \mathrm{~Hz}$, then that electrode will be pitch matched to a frequency that is very close to $500 \mathrm{~Hz}$ after the adaptation process is complete. In contrast, individuals with limited or incomplete adaptation may have PM scores that are closer to the SG function than to the FAT function.

There is evidence that some adaptation to the frequency-position function imposed by the CI does occur. Comparison of PM data obtained at the most apical electrode from different CI users at different times after initial stimulation (Francart et al, 2008; McDermott et al, 2009; Svirsky et al, 2012; Tan et al, 2012) suggests that pitch percepts elicited by electrical stimulation could change with experience and such electroacoustic PM can serve as a useful marker to determine whether a patient has fully adapted to the frequency-to-electrode map in his or her CI. Change in PM data over time is best assessed by examining longitudinal changes in the same individuals. Some longitudinal studies have found systematic PM changes as a function of experience (e.g., Reiss et al, 2007; 2008; 2014; 2015). On the other hand, Vermeire et al (2015) followed participants for a full year starting just before initial stimulation and found limited evidence of adaptation, but the difference between the SG and FAT curves was smaller than in other studies due to greater electrode insertion depth than in the Reiss et al's studies. This means that Vermeire et al's participants may have had less need to adapt than Reiss et al's participants.

The use of cross-sectional data obtained from experienced CI users is useful to get a sense of how many listeners show complete adaptation to the frequency-position function imposed by the CI. Some studies show good correspondence between PM scores and the FAT function and are therefore consistent with the possibility of complete adaptation. Vermeire et al (2008) studied this issue indirectly by conducting separate pitch-scaling experiments in the CI ear and the acoustically stimulated ear in 14 MED-EL (Durham, NC) CI users, after an average of 11 mo of experience. The derived PM curves were quite close to the FAT curve. In a more recent study, Vermeire et al (2015) studied PM between electrical stimulation at one electrode and acoustic tones in five unilateral MED-EL CI users with near-normal hearing in the contralateral ear. These participants showed a shift in their pitch matches toward the FAT, as they had more listening experience (although the shift failed to reach statistical significance in this case). After 12 mo of experience, four of five participants had PM results that followed the FAT curve closely. There was limited evidence of adaptation in their participants. This may have been due to the fact that all of Vermeire et al's participants used MED-EL electrodes, which are longer than those of other devices (Landsberger et al, 2015), possibly resulting in a smaller difference between the SG and FAT curves than in other studies. Again, Vermeire et al's participants may have had less need to adapt to a different frequency-place function than Reiss et al's participants.

However, there are results from other cross-sectional studies that suggest incomplete adaptation to the frequency-position function embodied in the FAT even after months of listening experience. Boëx et al (2006) tested six Clarion (Los Angeles, CA) CI users with at least 6 mo of device use. For four of them, the PM results were clearly lower frequency than 
the FAT; a fifth participant had PM results that were clearly higher than the FAT; and the last one presented a mixed picture depending on electrode location (higher frequency than the FAT for the two most apical ones, lower than the FAT for the others). Dorman et al (2007) also tested one MED-EL CI patient whose hearing in his unimplanted ear improved 15 mo after surgery for a CI. They reported his PM data were higher than the FAT for the two most apical electrodes and lower than the FAT for electrodes 4-11. Francart et al (2008) reported that only five of ten $\mathrm{CI}$ users pitch matched their most apical electrode to a frequency between 188 and $313 \mathrm{~Hz}$, which is the range assigned to that electrode in their speech processors. The other five $\mathrm{CI}$ users pitch matched that electrode to higher frequencies. In studies by Reiss et al $(2007 ; 2008)$, only 5 of the 12 CI users decreased their initial perceived pitch at the most apical electrode over time until it matched the FAT. The PM data perceived by the other CI users differed by as much as two octaves from the FAT (Reiss et al, 2008).

The present study aims to contribute to the conversation about pitch percepts caused by intracochlear electrodes, particularly when considered in conjunction with estimates of each electrode's insertion angle. In particular, it is hoped that the present results may help reconcile the differing results obtained in previous studies that combined PM with electrode location estimates, many of which were conducted using more deeply inserted electrodes than the ones used here.

\section{METHODS}

\section{Participants}

Nineteen postlingually deaf adults with CIs were initially screened to make sure they understood and could carry out the PM task. All but three of these participants passed the screening. Of the remaining 16 participants, 4 were implanted with the Advanced Bionics (AB; Valencia, $\mathrm{CA}$ ) device and 12 were implanted with Cochlear devices (Table 1). They were implanted at the Cochlear Implant Center, Department of Otolaryngology, New York University. All had some degree of residual acoustic hearing in their unimplanted ear as shown in Table 1 along with other demographic information.

\section{Procedure for Matching Pitch between Electric and Acoustic Stimuli}

Participants were first asked to adjust the intensity of the acoustic tone (presented to the unimplanted ear) to be perceived as "loud, but comfortable" and close to the perceived loudness elicited by the electrode to be tested, which was stimulated at the participant's maximum comfortable level. This was repeated for all electrodes to be tested. Stimuli were presented in alternating manner across ears, with a 500-msec pure tone presented acoustically to the unimplanted ear followed by $500 \mathrm{msec}$ of electrical stimulation to the implanted ear (see Figure 1). To obtain PM estimates for a given electrode, electrical stimulation was held constant while the frequency of the acoustical stimulation was adjusted using a slider on a computer screen until participants were satisfied that the pitch of the two stimuli was equivalent. They were also instructed that they should not indicate a match with a particular acoustic frequency without also having listened to both higher and lower frequencies. Participants were reminded to focus solely on the pitch attribute. To help participants focus on the pitch percepts produced by different electrodes, all electrodes were 
stimulated in turn before starting the experiment (after performing the intensity match described above), starting at the apical end of the array and continuing until the most basal end of the array.

PM was performed using as many of the eight most apical active electrodes in the array as time would allow. For each electrode, participants were required to complete six PM trials. The starting frequency of the acoustic tone was randomized from a range between 20 and $10000 \mathrm{~Hz}$ for each trial to assess the presence of nonsensory bias effects (Carlyon et al, 2010). For each electrode from each participant, clear outlier PM trials (those that were outside a $99.5 \%$ confidence interval of all trials for that electrode/participant combination) were discarded. The final pitch-matched value was the average of the remaining trials. Eight of 16 participants did not have any outliers. For the remaining eight participants, only one to three trials were discarded. Finally, we identified any electrode pairs whose PM values were antitonotopic by conducting one-way analyses of variance on each participant's PM values for different levels of the "electrode" variable, followed by post hoc comparisons for all pairs of electrodes at an overall significance level of 0.05 (Holm-Sidak method).

\section{Experimental Setup}

Pure tones were presented to the unimplanted ear via Sennheiser (Wedemark, Germany) HD580 headphones. Electrical stimulation was presented to the implanted ear via streaming hardware for Cochlear devices, and using direct audio connection for $\mathrm{AB}$ devices. For Cochlear devices, the electrical stimulation consisted of a pulse train delivered at the participant's everyday stimulation rate, pulse width, and maximum comfortable stimulation level (C-level). For AB devices, electrical stimulation was provided by presenting a 500msec tone whose frequency was the same as the center frequency of the analysis band associated with the electrode of interest. This was presented to the speech processor via direct audio connection (see Figure 1). Offline measurements made using an implant-in-abox confirmed that electrical stimulation was indeed centered very close to the desired electrode. Electrical stimulation patterns were recorded using a multichannel high-speed analog-to-digital converter in response to the tones that were used to drive the speech processor during the experiment. This was done separately (and with similar results) for all three stimulation strategies used by our participants with AB CIs, namely HiRes-P with Fidelity 120, HiRes-S with Fidelity 120, and HiRes-Optima-P. Figure 2 shows typical results for electrode 1 (Figure 2A) and for all other electrodes (ex-emplified in this case by electrode 3; Figure 2B). Vertical bars show the average stimulation level expressed as a percentage of electrical dynamic range, with zero corresponding to threshold and 100 corresponding to the maximum level programmed in the speech processor. For the tones at the center frequency of electrode 2 and higher the center of stimulation was almost exactly at the desired electrode (e.g., electrode 3 in Figure 2B). For electrode 1, however, which does not have a more apical neighboring electrode, the average place of stimulation was about $30 \%$ of the way between electrodes 1 and 2 (closer to electrode 1; see Figure 2A). This slight shift is taken into account in the analyses.

The acoustic tones for the unimplanted ear were amplified with a gain function prescribed using the National Acoustic Laboratory - Revised Profound fitting formula based on the 
participant's pure-tone audiogram (Byrne and Dillon, 1986; Byrne et al, 1990). All acoustic stimuli generated by our program were sampled at $22.05 \mathrm{kHz}$ with 16-bit resolution using a Sound Blaster Audigy SZ soundcard and amplified by a Sony amplifier, before they were presented to participants via a Sennheisser HD580 headphone. The acoustic tone was weighted by a trapezoidal window with a rise/fall of $20 \mathrm{msec}$ to prevent spectral splatter.

This setup was implemented in a program written in $\mathrm{C}++$. For Cochlear devices electrical stimulation was implemented using the Nucleus Implant Communicator research software library provided by Cochlear Ltd. (Denver, CO), and stimulation pulses were sent via a standard Freedom $^{\mathrm{TM}}$ (Denver, CO) speech processor. Nucleus Implant Communicator software allowed electrical stimulation to be delivered to the intracochlear electrode as specified by the programmer. Electrical stimulation for $\mathrm{AB}$ devices was delivered to the participant's implanted ear using a direct audio interface cable via a Harmony speech processor programmed with the participant's latest clinical map using SoundWave 2.2 ${ }^{\mathrm{TM}}$ (Valencia, CA).

\section{Nonsensory Biases Check}

Carlyon et al (2010) have shown that electroacoustic pitch comparisons can be strongly influenced by non-sensory biases arising from the range of acoustic stimuli presented, and have proposed two "checks" to rule out this possibility. One check for data obtained from pitch matches is that there should be no significant correlation between the starting frequency and final match across runs. Another requirement is that a sufficiently wide range of starting frequencies is used, and that the matches obtained converge onto a smaller range than that of the starting frequencies. More specifically, we used the criterion that the standard deviation of the final pitch-match values for a given electrode should be significantly smaller $(p<0.05)$ than that of the initial starting frequencies.

\section{Insertion Angle of Intracochlear Electrode}

The insertion angle of each electrode was estimated by using intraoperative plain radiograph images of participants' implanted cochlea (Landsberger et al, 2015; Svrakic et al, 2015) and based on methods described by earlier investigators (Marsh et al, 1993; Cohen et al, 1996; $\mathrm{Xu}$ et al, 2000). To evaluate objectively the insertion angles of the intracochlear electrodes in the array, several fixed reference points were used (see Figure 3). One reference line was drawn through the apex of the superior semicircular canal and the midpoint of the vestibule. The point where this line intersects the electrode lead is considered a good estimate of the round window location. Then a $0^{\circ}$ orientation line can be established by drawing through the estimated round window location and the estimated center point of the cochlear spiral. Finally, lines were drawn through the center of each electrode and the center point of the cochlear spiral. The angle between this line and the $0^{\circ}$ orientation line was measured as the insertion angle of the electrode in question.

\section{CFs along the $O C$ and SG at Measured Insertion Angles}

The CF of neurons in the OC and SG that might be stimulated by each electrode were estimated using Table 3 from the work of Stakhovskaya et al (2007), which provides CF estimates for different insertion angles. For example, that table shows that a $360^{\circ}$ angle 
corresponds to an OC mean frequency of $920 \mathrm{~Hz}$ and an SG mean frequency of $758 \mathrm{~Hz}$. We used linear interpolation to obtain angles that lie between angles listed in that table.

\section{RESULTS}

Figure 4 shows the PM data in 16 different panels, one for each participant. In each panel, the units for both axes are Hz. The $x$ axis indicates the center frequency of the analysis band allocated to the eight most apical active electrodes as specified in the participant's clinical FAT. Cochlear and AB systems conventionally number their electrodes in different ways: the former goes base to apex and the latter goes apex to base. For simplicity, all electrode arrays in this report have been numbered starting at the apex. The $y$ axis has a different meaning for each of the four curves found in each panel. The main result of the study is shown by black squares connected with black lines, indicating the average acoustic frequency that was pitch matched by each participant to each one of the eight most apical electrodes. The two green dotted lines indicate the upper and lower range of pitch-matched frequencies for each electrode. The second curve (which we named "FAT curve" and indicated by red asterisks) shows the center frequency of the analysis band for each electrode in both the $x$ and $y$ axes. In other words, the line formed by the red asterisks would be a diagonal if both axes used the same scale. The third curve with magenta right-pointing triangles indicates the mean $\mathrm{CF}$ along the OC estimated at each electrode location, based on Greenwood's equation. Finally, the fourth curve is formed by blue left-pointing triangles that indicate the mean $\mathrm{CF}$ along the SG estimated at each electrode location using the angular location of each electrode and Table 3 from the work of Stakhovskaya et al (2007). Let us call this the "SG curve." Note that some participants' radiographic images were not available for insertion angle measurement (Participants 2, 5, 15, and 16), and therefore the OC and SG curves were not included in the corresponding panels. The red ovals in some panels indicate electrodes where the participant's PM trials failed the nonsensory bias checks (Carlyon et al, 2010). The gray-shaded region indicates the frequency range where the participant's residual hearing threshold in the nonimplanted ear is between 40 and $70 \mathrm{~dB}$ HL. Any frequencies above the gray-shaded region along the $y$ axis indicate thresholds poorer than $70 \mathrm{~dB} \mathrm{HL}$. It is likely that PM frequencies falling in this region are less meaningful than those obtained at lower frequencies. Participants 8 and 10 do not have a gray-shaded region in their graphs because all their thresholds within the frequency range of interest were better than $40 \mathrm{~dB}$ HL. The duration of CI use at the time of testing and the depth of insertion of the most apical electrode (when available) are indicated next to the participant number at the top of each panel.

The sixteen panels of Figure 4 paint a picture of significant individual differences across participants, and they have been organized according to the type of result. The main analysis in the present study consists of comparing the PM curves to the SG and FAT curves. The SG curve represents one estimate of the frequency-position function that was valid when the listener had normal hearing. The FAT curve represents the frequency-position function that the CI attempts to impose. Complete coincidence between a CI user's PM curve and the FAT curve would indicate that the CI user has adapted completely to the frequency-position function imposed by the CI, whereas a PM function that is close to the SG curve would suggest that the CI user has not been successful in remapping his or her natural frequency- 
position function after implantation. Results for Participants 1 and 2 are consistent with complete adaptation to the new frequency-position function imposed by the CI, as the PM and FAT curves overlap almost perfectly, particularly when considering the maximum and minimum PM results for each electrode (green lines). Participants 3, 4, 5, 6, and 7 show near-complete adaptation to the FAT curve, with the sole exception of the most apical electrode or electrode 2. The PM curves for Participants 8, 9, and 10 are roughly halfway between the SG and the FAT curves, consistent with incomplete adaptation to the FAT curve. Note, however, that Participant 9 was tested only 2 days after initial stimulation. This is much earlier than for all other participants, and this limited amount of time may have been insufficient to achieve much adaptation. Only 2 of 16 participants (Participants 11 and 12) had PM curves that were closer to the SG curve than to the FAT curve. Note that Participant 12 is the oldest in the present sample, 82 yr 8 mo at the time of testing. Participant 11 is one of the oldest in the sample, $76.5 \mathrm{yr} 6 \mathrm{mo}$, and also one of the least experienced participants at only 3 mo after initial stimulation. Participants 13, 14, and 15 were similar to Participants 1-10 in that their PM curves were closer to the FAT curve than to the SG curve. However, their PM curves were largely lower in frequency than the FAT curves rather than higher. In the case of Participant 13, this result may have been influenced by his limited residual hearing. Participant 14 had the most deeply implanted electrode array: at $453^{\circ}$ it was $61^{\circ}$ deeper than the second deepest insertion in the present sample. Finally, Participant 16 had an unusual pattern of residual hearing and for the most part was unable to perform the PM task reliably.

Despite individual differences, the first overall trend that emerges from these data is that with the exception of only one participant, PM curves have lower frequency values than the SG curve. To the extent that the SG curve represents a natural frequency-position function, this finding would indicate that most of these CI users have shown some degree of adaptation away from that function, in the direction of the FAT function. For almost half of the participants, this adaptation to the FAT curve was complete or almost complete (with the exception of the most apical electrode or two). A second overall trend in the data is that the PM curves are, on average, somewhat higher in frequency than the FAT curves, suggesting that adaptation to the FAT curves has not been complete. In this case, the exceptions are Participants 14 and 15 (and possibly Participant 13).

The preceding observations were quantified in Table 2. Columns 2-4 refer to data from the four most apical electrodes and columns 5-7 show the corresponding data for the four relatively more basal electrodes, when available. The first column within each of those two ranges shows the mean difference in octaves between the PM curve and the SG curve, the second column indicates the mean difference in octaves between the PM curve and the FAT curve, and the third column indicates whether the PM curve is closer to the SG curve or the FAT curve. A negative sign indicates that the PM frequency is lower than the corresponding frequency in the comparison curve. The last row of Table 2 shows values averaged across all participants. Data in the table bear out the observations made based on examination of the plots shown in Figure 4. First, examination of columns 4 and 7 show that for both subsets of electrodes the PM curves are closer to the FAT curve than to the SG curve. This happens both for the apical electrodes ( -1.21 octaves versus 0.2 octaves) and for the next set of electrodes ( -0.9 octaves versus 0.27 octaves). This result is statistically significant as 
confirmed by matched pairs $t$ test comparisons of the absolute values of mean differences ( $p$ $<0.001$ for electrodes $1-4$ and $p<0.01$ for electrodes 5-8). Second, the negative values of almost all numbers in columns 2 and 5 show that the PM curves have lower values than the SG curve. Finally, the average values in columns 3 and 6 show that adaptation to the FAT curve has not been complete across the board.

Additional evidence for the idea that some CI patients show evidence of complete adaptation while others do not is shown in Figure 5, which shows PM data for the most apical electrode in Cochlear patients as a function of time after initial stimulation. The figure includes data from the current study and two previous studies (Francart et al, 2008; McDermott et al, 2009). The $y$ axis shows acoustic frequency pitch matched by each participant, and the $x$ axis shows the time after initial stimulation (in a logarithmic scale) when the PM was conducted. The upper and lower boundaries of the analysis band associated with the most apical electrode are indicated by the two horizontal dashed lines. In Cochlear devices, the analysis band is between 188 and $313 \mathrm{~Hz}$ with a center frequency at $250 \mathrm{~Hz}$. The blackfilled circles indicate the PM data from the current study; the gray-filled circles and the unfilled circles indicate the PM data from the two other studies as stated in the figure legend. Five of the McDermott et al's participants were tested before they had any listening experience with their $\mathrm{CI}$ and they showed substantial basalward shift (which is defined as having PM frequencies that are higher than the center frequency of the corresponding filter). This result is consistent with the average location of the most apical electrode for users of Cochlear devices, which is not deep enough to stimulate neurons whose CF is $250 \mathrm{~Hz}$ (Landsberger et al, 2015). In contrast, the 32 participants with several months or years of experience showed much smaller amounts of basalward shift than the five participants tested upon initial stimulation by McDermott et al (2009). Twelve of them showed no basalward shift at all, meaning that the average frequency that was pitch matched to the most apical electrode fell within the corresponding filter band of 188-313 Hz. Many of the other participants had PM values that were very close to the $188-313 \mathrm{~Hz}$ band.

On the other hand, many pitch-match frequencies showed incomplete adaptation to the FAT used every day by the participants. This can be appreciated in Figure 5, where two of our participants, two from McDermott et al, and one from Francart et al, had pitch-match frequencies close to $500 \mathrm{~Hz}$, almost one full octave above the center frequency of the corresponding filter (which is $250 \mathrm{~Hz}$, as pointed out above). This can also be appreciated in many of the PM curves from Figure 4. Even the participants whose pitch-match frequencies were very reliable, consistent, and closest to the FAT curve had some electrodes with evidence of incomplete adaptation. Finally, even though the data were largely tonotopic (more basal electrodes were almost always pitch matched to higher frequencies than more apical electrodes), there were even a few cases where PM revealed statistically significant antitonotopic percepts. Some examples include electrodes 2 and 3, 2 and 5, and 4 and 5 for Participant 9; electrodes 5 and 6 for Participant 11; and electrodes 7 and 8 for Participant 12.

\section{DISCUSSION}

The present dataset shows important individual differences but also some overall trends. Common trends will be discussed first. As a group, PM curves had lower frequency values 
than the SG curve (with Participant 12 as the sole exception) and were much closer to the FAT curve than to the SG curve for most participants (with Participants 11 and 12 being the exceptions). This is consistent with the possibility that initially, CI percepts were subject to basalward shift (as suggested by Svirsky et al, 2001; 2004; Reiss et al, 2007; McDermott et al, 2009; Landsberger et al, 2015), but the PM changed as a function of experience, with the final PM curve being much closer to the FAT curve than to the SG curve. This is also suggested by the average pitch-matched frequencies obtained from our less experienced participants: 11 (3 mo after initial stimulation) and 9 ( 2 days after initial stimulation).

Another trend in the data, consistent with the preceding explanation, is that the PM curves were, on average, somewhat higher in frequency than the FAT curves, suggesting that adaptation to the FAT curves was not complete for many participants. Although there were individual exceptions, the trends that were summarized and quantified in Table 2 are clear: PM curves were $~ 0.9-1.2$ octaves lower than SG curves and only $\sim 0.2-0.3$ octaves higher than the FAT curves. Overall, about half of the participants ( 7 of the 15 who could do the task reliably) had PM curves that were either identical or very close to the FAT curve. The latter showed similar small departures from the FAT curve: in all five cases (Participants 37) there was some basalward shift in the most apical electrode.

It is particularly interesting to examine the most apical electrode of Nucleus CI users, allowing the comparison between our data and those of two previous studies (Francart et al, 2008; McDermott et al, 2009; see Figure 5). Five of McDermott et al's participants were tested before they had any listening experience with their CI. They showed a substantial basalward shift in the pitch percept, which is consistent with the average location of the most apical electrode for users of Cochlear devices, whose physical insertion depth may not address direct stimulation of neurons with $250 \mathrm{~Hz}$ CF (Landsberger et al, 2015). After several months or years of experience, 9 of 22 experienced CI users displayed little to no frequency shift: the acoustic pitch match fell within the frequency range assigned to that electrode (188-313 Hz). In contrast, 13 experienced CI users still displayed different amounts of basalward shift, as the acoustic pitch match was higher than $313 \mathrm{~Hz}$. This can be seen in Figure 5, where five of the experienced users had pitch-match frequencies that were not only > $313 \mathrm{~Hz}$ but also were close to $500 \mathrm{~Hz}$, almost one full octave above the center frequency of the corresponding filter (i.e., $250 \mathrm{~Hz}$ ). Incomplete adaptation can also be appreciated in many of the PM curves from Figure 4. These results are consistent with other examples of incomplete adaptation found in previous published studies, whether they used PM (Reiss et al, 2007; 2008; 2015) or other methods (Harnsberger et al, 2001; Svirsky et al, 2004; 2015).

Finally, it is of interest to discuss apparent discrepancies among studies of electroacoustic PM. Many of these studies found PM scores that were generally well below those that would be predicted based on a corrected Greenwood function (e.g., Boëx et al, 2006; Dorman et al, 2007; Reiss et al, 2007; McDermott et al, 2009; Green et al, 2012; the present study); others found good correspondence between PM scores and Greenwood function numbers (Carlyon et al, 2010, and Schatzer et al, 2014, at least for electrodes in the first cochlear turn). Another discrepancy is that some studies found evidence of significant changes in pitch matches over time or at least across participants, as a function of experience with the implant 
(e.g., Reiss et al, 2007; McDermott et al, 2009), whereas other studies did not find such shifts over time (Vermeire et al, 2015). Carlyon et al's results are also consistent with the latter two studies in that the only participant who showed PM changes as a function of time was one whose electrode array actually moved over time, in a direction that was consistent with the PM changes.

One possible way to account for some of these discrepancies is to postulate that the experience-dependent changes under discussion may happen only in response to ecological demands. In other words, listeners with deeper electrode insertions (such as those in the Vermeire et al's 2015 and Schatzer et al's 2014, studies) may have had lower levels of initial basalward mismatch than participants in other studies (such as ours) who had shallower electrode insertions, and therefore, there may have been no need for this type of experiencedependent change. Carlyon et al's participants did not have such deep insertions but they did have normal hearing in the contralateral ear, so they were able to understand speech even without any contributions from the implanted ear. Again, there was no compelling need to adapt to the Cl's frequency-position function in this case. One would expect that listeners who initially suffer from basalward shift and adapt in response to it, will have PM curves that become lower than the SG curve or the Greenwood function after the adaptation process. In contrast, listeners whose PM curves do not change significantly as a function of experience after implantation (either because they do not have frequency mismatch or because they lack the ecological motivation to adapt) would be expected to have PM curves that are closer to the SG curve or the Greenwood function.

Taken together, the present data combined with other studies of electroacoustic PM provide strong support for the hypothesis that the adult brain is plastic in response to experience deemed relevant by the listener, and that this plasticity allows postlingually deaf listeners to overcome the changes in peripheral frequency-position functions that may be imposed by cochlear implantation. However, this plasticity is not always sufficient to overcome large frequency-position mismatches. These findings are likely to help guide future development of intracochlear electrodes and speech processing strategies for CIs, as well as the clinical management of CI users.

\section{Acknowledgments}

The authors are grateful to Arlene Neuman, David Landsberger, Annette Zeman, Mahan Azadpour, Matthew Fitzgerald, Ksenia Prosolovich, Elad Sagi, Elizabeth Glassman, Keena Seward, and Xiang Zhou for their advice and cooperation during testing of participants with CIs, and for important comments on the manuscript. Two anonymous reviewers also made suggestions that significantly enhanced the manuscript. Finally, the authors also thank the participants for their help.

Author C.T. was supported by NIH/NIDCD grant number 1K25DC010834-01; author M.S. was supported by NIH/ NIDCD grant numbers R01-DC03937 and R01-DC011329; author B.M. was supported by a PSC-CUNY grant. This research was also supported by a research contract from Cochlear Americas (P.I.: J. Thomas Roland Jr.).

\section{Abbreviations}
AB
Advanced Bionics
CF
characteristic frequency 
CI cochlear implant

FAT frequency allocation table

OC organ of Corti

PM pitch matching

SG spiral ganglion

\section{References}

Boëx C, Baud L, Cosendai G, Sigrist A, Kós MI, Pelizzone M. Acoustic to electric pitch comparisons in cochlear implant subjects with residual hearing. J Assoc Res Otolaryngol. 2006; 7(2):110-124. [PubMed: 16450213]

Byrne D, Dillon H. The National Acoustic Laboratories' (NAL) new procedure for selecting the gain and frequency response of a hearing aid. Ear Hear. 1986; 7(4):257-265. [PubMed: 3743918]

Byrne D, Parkinson A, Newall P. Hearing aid gain and frequency response requirements for the severely/profoundly hearing impaired. Ear Hear. 1990; 11(1):40-49. [PubMed: 2307302]

Carlyon RP, Macherey O, Frijns JHM, Axon PR, Kalkman RK, Boyle P, Baguley DM, Briggs J, Deeks JM, Briaire JJ, Barreau X, Dauman R. Pitch comparisons between electrical stimulation of a cochlear implant and acoustic stimuli presented to a normal-hearing contralateral ear. J Assoc Res Otolaryngol. 2010; 11(4):625-640. [PubMed: 20526727]

Cohen LT, Xu J, Xu SA, Clark GM. Improved and simplified methods for specifying positions of the electrode bands of a cochlear implant array. Am J Otol. 1996; 17(6):859-865. [PubMed: 8915414]

Dorman MF, Spahr T, Gifford R, Loiselle L, McKarns S, Holden T, Skinner M, Finley C. An electric frequency-to-place map for a cochlear implant patient with hearing in the nonimplanted ear. J Assoc Res Otolaryngol. 2007; 8(2):234-240. [PubMed: 17351713]

Fitzgerald MB, Sagi E, Jackson M, Shapiro WH, Roland JT Jr, Waltzman SB, Svirsky MA. Reimplantation of hybrid cochlear implant users with a full-length electrode after loss of residual hearing. Otol Neurotol. 2008; 29(2):168-173. [PubMed: 18165793]

Francart T, Brokx J, Wouters J. Sensitivity to interaural level difference and loudness growth with bilateral bimodal stimulation. Audiol Neurotol. 2008; 13:309-319.

Green T, Faulkner A, Rosen S. Frequency selectivity of contralateral residual acoustic hearing in bimodal cochlear implant users, and limitations on the ability to match the pitch of electric and acoustic stimuli. Int J Audiol. 2012; 51(5):389-398. [PubMed: 22201528]

Greenwood DD. A cochlear frequency-position function for several species-29 years later. J Acoust Soc Am. 1990; 87(6):2592-2605. [PubMed: 2373794]

Harnsberger JD, Svirsky MA, Kaiser AR, Pisoni DB, Wright R, Meyer TA. Perceptual "vowel spaces" of cochlear implant users: implications for the study of auditory adaptation to spectral shift. $\mathrm{J}$ Acoust Soc Am. 2001; 109(5 Pt 1):2135-2145. [PubMed: 11386565]

Landsberger DM, Svrakic M, Roland JT Jr, Svirsky M. The relationship between insertion angles, default frequency allocations, and spiral ganglion place pitch in cochlear implants. Ear Hear. 2015; 36(5):e207-e213. [PubMed: 25860624]

Marsh MA, Xu J, Blamey PJ, Whitford LA, Xu SA, Silverman JM, Clark GM. Radiologic evaluation of multichannel intracochlear implant insertion depth. Am J Otol. 1993; 14(4):386-391. [PubMed: 8238277]

McDermott H, Sucher C, Simpson A. Electro-acoustic stimulation. Acoustic and electric pitch comparisons. Audiol Neurootol. 2009; 14(1 Suppl):2-7. [PubMed: 19390169]

Reiss LA, Gantz BJ, Turner CW. Cochlear implant speech processor frequency allocations may influence pitch perception. Otol Neurotol. 2008; 29(2):160-167. [PubMed: 18025998]

Reiss LA, Ito RA, Eggleston JL, Liao S, Becker JJ, Lakin CE, Warren FM, McMenomey SO. Pitch adaptation patterns in bimodal cochlear implant users: over time and after experience. Ear Hear. 2015; 36(2):e23-e34. [PubMed: 25319401] 
Reiss LAJ, Turner CW, Erenberg SR, Gantz BJ. Changes in pitch with a cochlear implant over time. J Assoc Res Otolaryngol. 2007; 8(2):241-257. [PubMed: 17347777]

Reiss LA, Turner CW, Karsten SA, Gantz BJ. Plasticity in human pitch perception induced by tonotopically mismatched electro-acoustic stimulation. Neuroscience. 2014; 256:43-52. [PubMed: 24157931]

Schatzer R, Vermeire K, Visser D, Krenmayr A, Kals M, Voormolen M, Van de Heyning P, Zierhofer C. Electric-acoustic pitch comparisons in single-sided-deaf cochlear implant users: frequencyplace functions and rate pitch. Hear Res. 2014; 309:26-35. [PubMed: 24252455]

Stakhovskaya O, Sridhar D, Bonham BH, Leake PA. Frequency map for the human cochlear spiral ganglion: implications for cochlear implants. J Assoc Res Otolaryngol. 2007; 8(2):220-233. [PubMed: 17318276]

Svirsky MA, Fitzgerald MB, Neuman A, Sagi E, Tan CT, Ketten D, Martin B. Current and planned cochlear implant research at New York University Laboratory for Translational Auditory Research. J Am Acad Audiol. 2012; 23(6):422-437. [PubMed: 22668763]

Svirsky MA, Fitzgerald MB, Sagi E, Glassman EK. Bilateral cochlear implants with large asymmetries in electrode insertion depth: implications for the study of auditory plasticity. Acta Otolaryngol. 2015; 135(4):354-363. [PubMed: 25719506]

Svirsky MA, Silveira A, Neuburger H, Teoh S-W, Suárez H. Long-term auditory adaptation to a modified peripheral frequency map. Acta Otolaryngol. 2004; 124(4):381-386. [PubMed: 15224858]

Svirsky MA, Silveira A, Suarez H, Neuburger H, Lai TT, Simmons PM. Auditory learning and adaptation after cochlear implantation: a preliminary study of discrimination and labeling of vowel sounds by cochlear implant users. Acta Otolaryngol. 2001; 121(2):262-265. [PubMed: 11349792]

Svrakic M, Friedmann DR, Berman PM, Davis AJ, Roland JT Jr, Svirsky MA. Measurement of cochlear implant electrode position from intraoperative post-insertion skull radiographs: a validation study. Otol Neurotol. 2015; 36(9):1486-1491. [PubMed: 26375970]

Tan C-T, Guo B, Martin BA, Svirsky MA. Behavioral and physiological measure for pitch matching between electrical and acoustical stimulation in cochlear implant patients. Proc Mtgs Acoust. 2012; 15:050003.

Toner J, McAnallen C, Proops D, et al. UK Cochlear Implant Study Group. Criteria of candidacy for unilateral cochlear implantation in postlingually deafened adults II: cost-effectiveness analysis. Ear Hear. 2004; 25(4):336-360. [PubMed: 15292775]

Xu J, Xu SA, Cohen LT, Clark GM. Cochlear view: postoperative radiography for cochlear implantation. Am J Otol. 2000; 21(1):49-56. [PubMed: 10651435]

Vermeire K, Landsberger DM, Schleich P, Van de Heyning PH. Multidimensional scaling between acoustic and electric stimuli in cochlear implant users with contralateral hearing. Hear Res. 2013; 306:29-36. [PubMed: 24055624]

Vermeire K, Landsberger DM, Van de Heyning PH, Voormolen M, Kleine Punte A, Schatzer R, Zierhofer C. Frequency-place map for electrical stimulation in cochlear implants: change over time. Hear Res. 2015; 326:8-14. [PubMed: 25840373]

Vermeire K, Nobbe A, Schleich P, Nopp P, Voormolen MH, Van de Heyning PH. Neural tonotopy in cochlear implants: an evaluation in unilateral cochlear implant patients with unilateral deafness and tinnitus. Hear Res. 2008; 245(1-2):98-106. [PubMed: 18817861] 


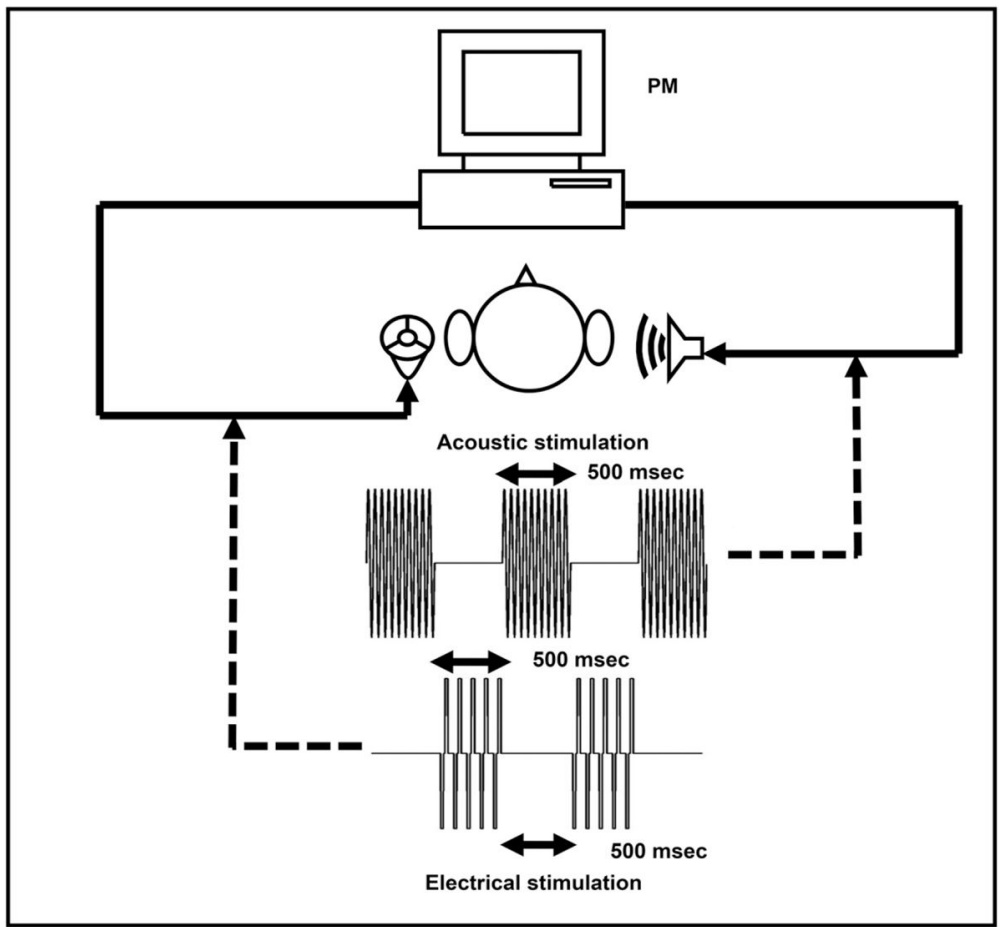

Figure 1.

Experimental setup for electroacoustic PM by participants with CIs. 

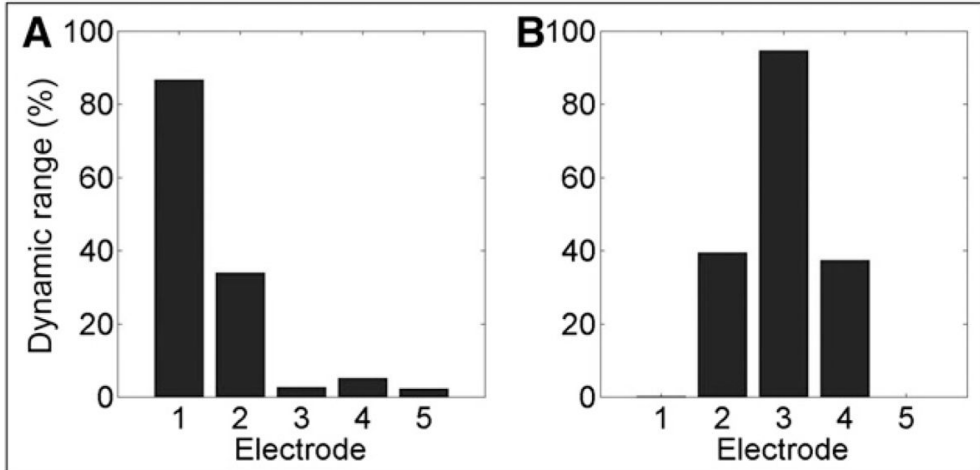

Figure 2.

Electrical output of the $\mathrm{AB} \mathrm{CI}$ as a percentage of dynamic range for each electrode, when the input acoustic tone is (A) at the center frequency of electrode 1 and (B) electrode 3. 


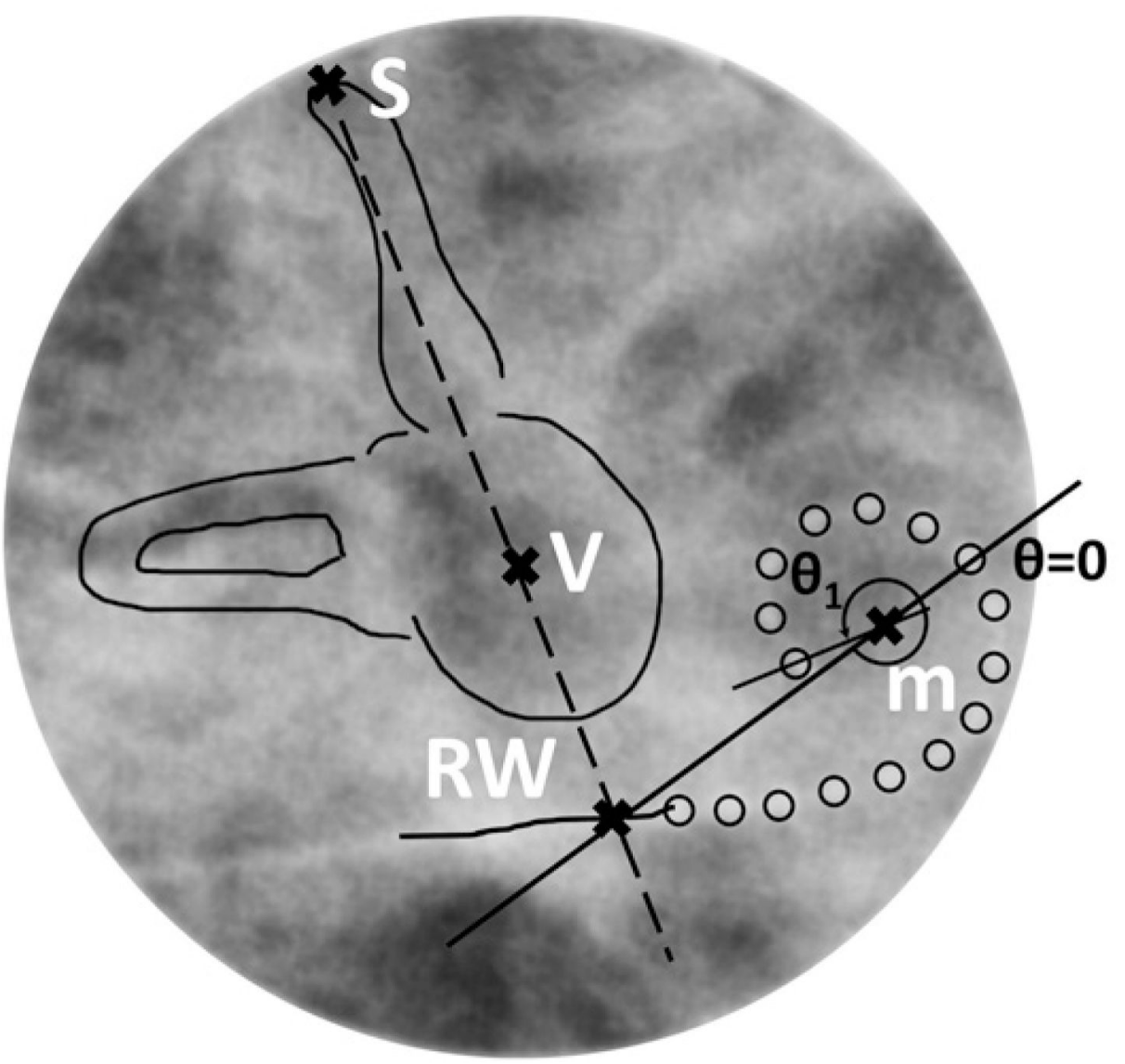

Figure 3.

Fixed reference points and lines of a postoperative modified Stenver's view plain radiograph image of an implanted cochlea for estimating electrode insertion angles. $\mathrm{m}=$ modiolus; RW = round window; $\mathrm{S}=$ superior semicircular canal; $\mathrm{V}=$ vestibule. 

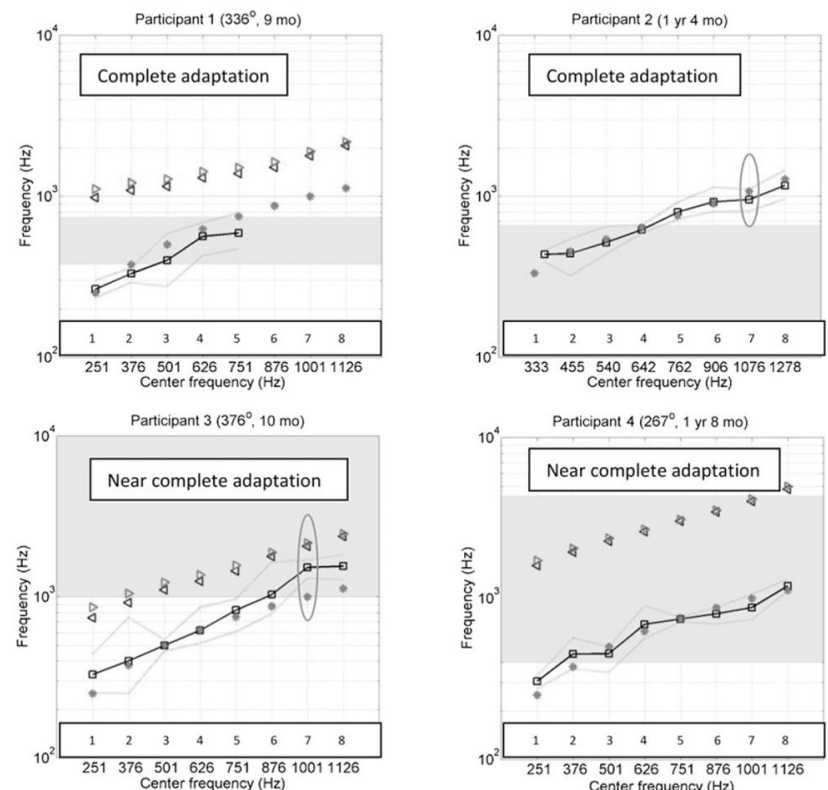

$10^{4} \quad$ Participant $5(8 \mathrm{yr} 1 \mathrm{mo})$
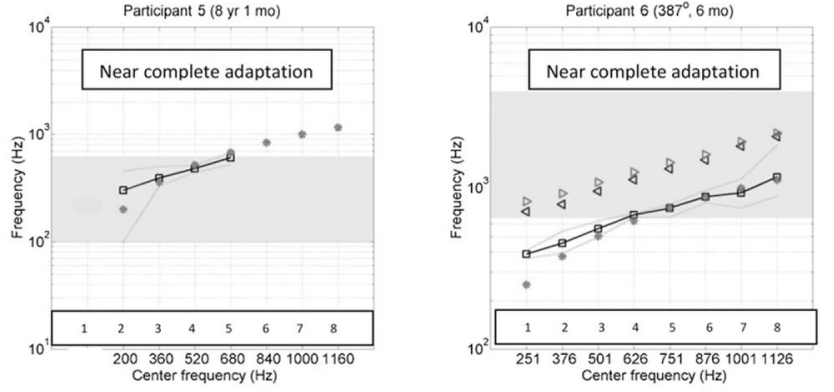

$\begin{array}{llllll}10^{2} & 5131376 \quad 501626 & 751876 & 10011126 \\ \text { Center frequency } & (\mathrm{Hz})\end{array}$

$10^{4} \quad$ Participant $7\left(334^{\circ} .7 \mathrm{yr} 11 \mathrm{mo}\right)$

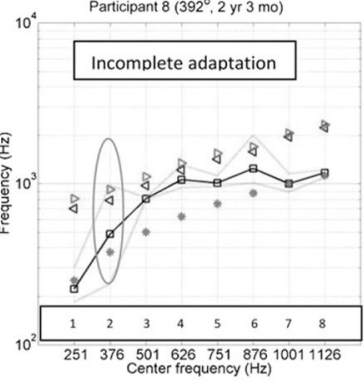



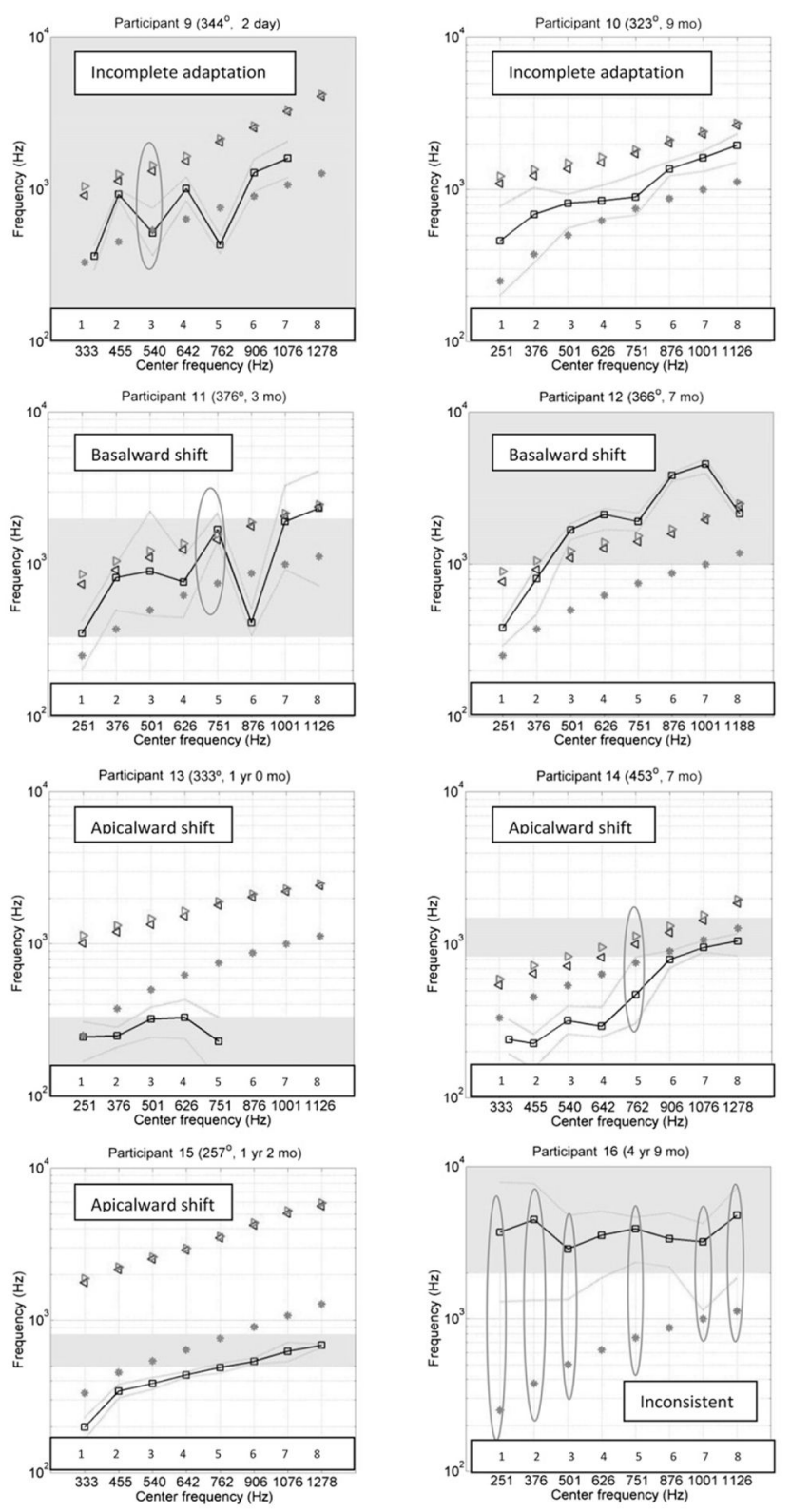

Figure 4.

Individual PM curves for all participants (black lines connecting square symbols show the average and green lines without symbols show the whole range of PM numbers) compared to frequency-position functions along the SG (blue left-pointing triangles) and OC (magenta right-pointing triangles). Also shown is the frequency-position function of CI stimulation (red asterisks), which results from a combination of each individual's FAT and electrode location. Ovals indicate electrodes with unreliable PM. (This figure appears in color in the online version of the article.) 


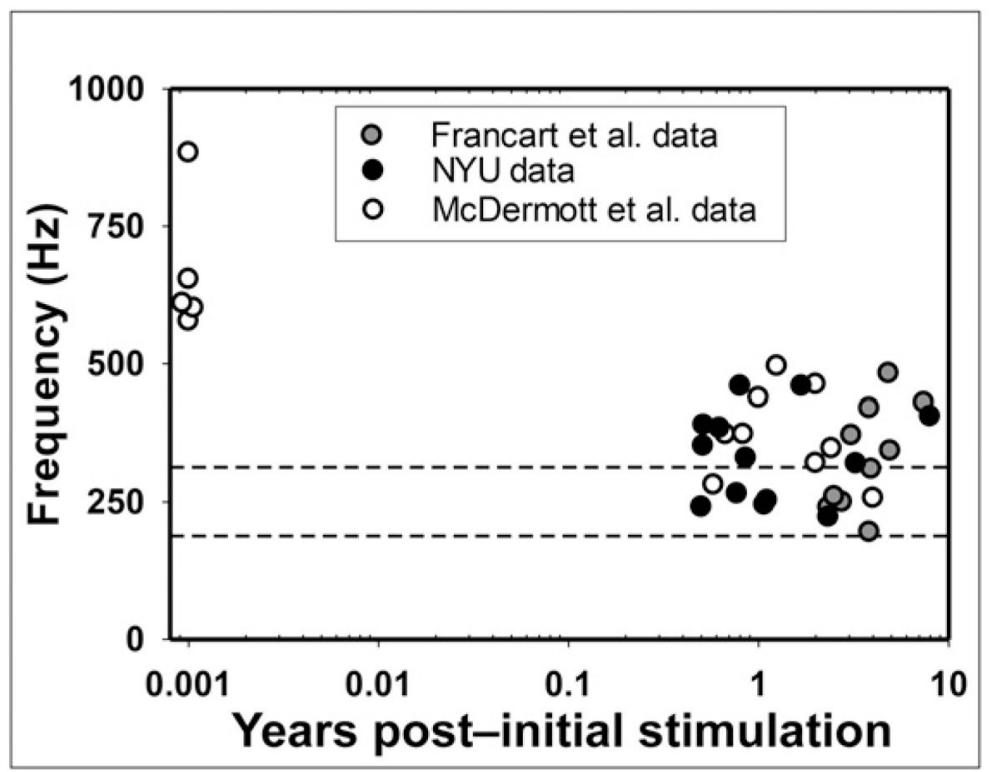

Figure 5.

PM data for electrode 22 of Nucleus users in the present study (black circles) and two other studies, as a function of time after initial stimulation. In all cases, electrode 22 was associated with a center frequency of $250 \mathrm{~Hz}$ and a frequency range of 188-313 Hz (indicated by horizontal dotted lines). NYU = New York University. 


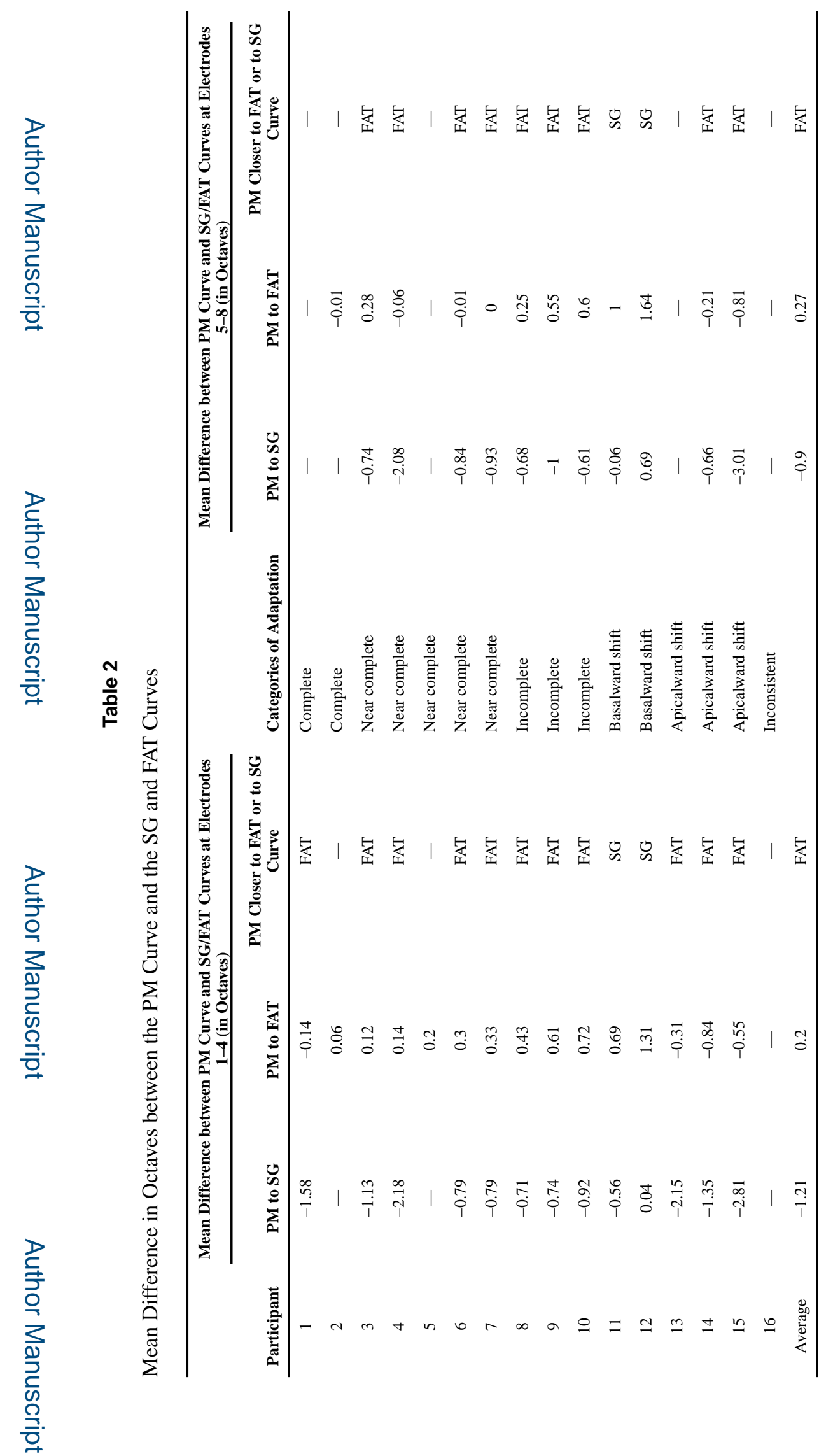

J Am Acad Audiol. Author manuscript; available in PMC 2017 May 17. 